\title{
The Development of Silica Hydride Stationary Phases for High-Performance Liquid Chromatography from Conception to Commercialization
}

\author{
Maria Matyska and Joseph Pesek* \\ Department of Chemistry, San Jose State University, San Jose, CA 95192, USA; maria.matyska-pesek@sjsu.edu \\ * Correspondence: joseph.pesek@sjsu.edu; Tel.: +1-408-924-4950
}

Received: 24 February 2019; Accepted: 24 April 2019; Published: 21 May 2019

check for updates

\begin{abstract}
The development of a stationary phase material for high-performance liquid chromatography based on a surface of silica hydride as opposed to silanols on ordinary silica is discussed including synthetic approaches, characterization, and applications. There are several synthetic approaches available to create a silica hydride surface. Modification of the $\mathrm{Si}-\mathrm{H}$ moiety on the silica surface can be accomplished through the use of a hydrosilation reaction. Both the intermediate silica hydride and the material modified with an organic moiety can be characterized by a number of spectroscopic as well as a variety of other methods. Further insights into the retention mechanism are provided through chromatographic measurements. The ultimate utility of any chromatographic stationary phase material is determined by its success in solving challenging analytical problems. A broad range of applications is reviewed to illustrate the versatility and usefulness of silica hydride-based stationary phases.
\end{abstract}

Keywords: hydride surface; silicon-carbon bond; aqueous normal phase; retention mechanism

\section{Introduction}

The fundamental properties of silica as a support material for high performance liquid chromatography were described by Unger [1] quite some time ago. The information provided with respect to silica in this monograph is essentially unchanged after several decades. Among these properties, two of the most significant are the presence of silanols on the surface and the ability to modify these entities with a number of known chemical reactions. The former defines the surface as a strongly polar medium, while the latter provides a means of modifying this polarity with suitable organic moieties in order to produce a stationary phase of the desired chemical properties. The most common type of chemical modification that is used for virtually all commercially available products for HPLC (High Performance Liquid Chromatography) based on silica, is organosilanization. There are two possible approaches for this reaction scheme as shown below:

MONOMERIC BONDING

$\mathrm{Si}-\mathrm{OH}+\mathrm{X}-\mathrm{Si} \mathrm{R}_{2}^{\prime} \mathrm{R} \longrightarrow \mathrm{Si}-\mathrm{O}-\mathrm{Si} \mathrm{R}_{2}^{\prime} \mathrm{R}+\mathrm{HX}$

$\mathrm{X}$-halide and R-alkyl

POLYMERIC BONDING

$\mathrm{Si}-\mathrm{OH}+\mathrm{X}_{3}-\mathrm{Si}-\mathrm{R} \longrightarrow \mathrm{Si}-\mathrm{O}-\mathrm{Si}-\mathrm{R}+3 \mathrm{HX}$

In the case of monomeric bonding, an organosilane compound with one reactive group reacts with the silanol on the silica surface to produce a single point of attachment via silicon-oxygen-silicon-carbon bonds. For polymeric bonding, an organosilane compound with three reactive groups forms a bond to the surface but also crosslinks with adjacent organosilane reagents to form a polymeric network on the 
surface. While the latter usually leads to higher carbon loads, i.e., more organic moieties bonded to the surface, the reaction is difficult to control and often results in considerable variation in the stationary phase from batch to batch. More reproducible materials are made from the monomeric bonding process, and thus it is the approach used for a significant number of commercial HPLC stationary phases based on silica.

A drawback of the organosilanization approach is the $\mathrm{Si}-\mathrm{O}-\mathrm{Si}-\mathrm{C}$ linkage at the surface between the silica support material and the bonded organic moiety. While stable in a variety of oregano-aqueous mobile phases it has limitations based on $\mathrm{pH}$ stability. At low $\mathrm{pH}$, the $\mathrm{O}-\mathrm{Si}-\mathrm{C}$ point of attachment is subject to hydrolysis while at higher $\mathrm{pH}$ the support silica is prone to a dissolution process. Thus a goal for improvement of stationary phase stability is to fundamentally alter this bonding reaction and to produce a direct silicon-carbon bond at the surface. An early approach to achieve this goal was the use of a two-step process involving a chlorination reaction followed by either Grignard or organolithium attachment to the surface [2] as shown below:

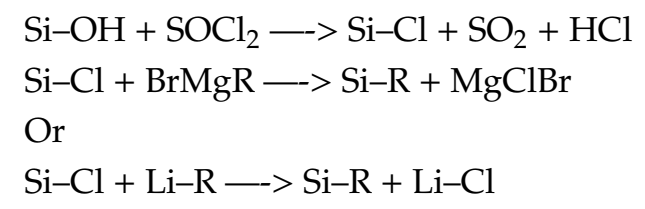

While this reaction scheme achieves the desired result of having a direct silicon-carbon bond between the silica surface and the attached organic moiety, it has some significant drawbacks. The reaction with thionyl chloride produces a material that is hydrolytically unstable. Thus, the synthesis must be done under scrupulously dry conditions or otherwise the chlorinated silica reverts back to the original material. In the second reaction with a Grignard reagent or an organolithium compound, the by-products are salts that can easily be trapped on the stationary phase surface and thus, result in erratic chromatographic behavior. Thus, while feasible, this is not a practical approach to make a commercially viable stationary phase based on a material with a direct bond between the surface and the organic moiety.

\section{Evolution of Silica Hydride Stationary Phases}

In the late 1980s and early 1990s, a new reaction protocol was developed in an attempt to use silica as a support material and having the organic moiety attached through a direct silicon-carbon bond [3-5]. This synthetic approach also involves a two-step process but is considerably less stringent with respect to reaction conditions than the previously described chlorination/Grignard or organolithium method. The first step is to change the surface morphology from one that is dominated by silanols to another reactive site for the attachment of organic groups. This goal is achieved by reacting silica with a trifunctional silane so that through controlled polymerization a monolayer is formed on the surface where the new functionality is an $\mathrm{Si}-\mathrm{H}$ group as shown below.

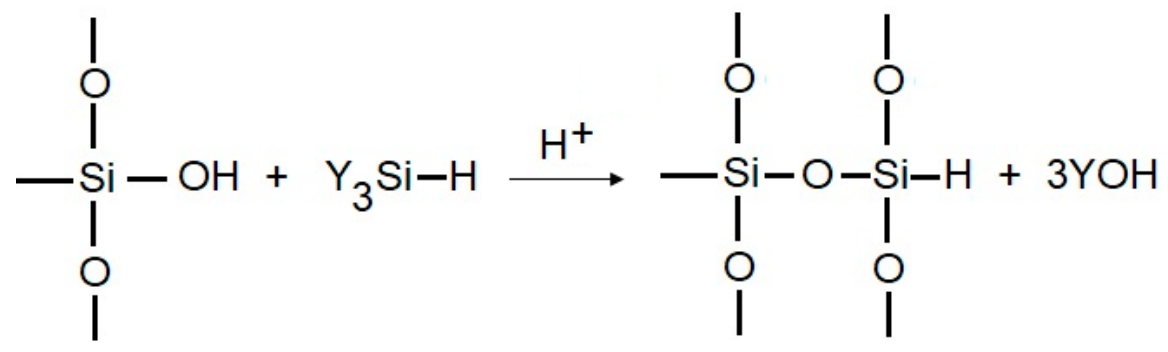

In this case, the surface of ordinary silica is transformed from a very polar medium to the slightly hydrophobic character of silica hydride. This has profound effects with respect to the chromatographic capabilities of silica hydride that will be described later in this review. The attractive feature of ordinary silica is that the surface could be modified by chemical reactions, such as those described above. 
This made it possible to tailor the surface, and hence the chromatographic properties, from the strong polar and adsorptive character of silica to quite hydrophobic when long chain aliphatic moieties are bonded to the surface.

However, silica hydride can be modified just as easily as ordinary silica through the use of a well-characterized reaction referred to hydrosilation. The general process is shown below.

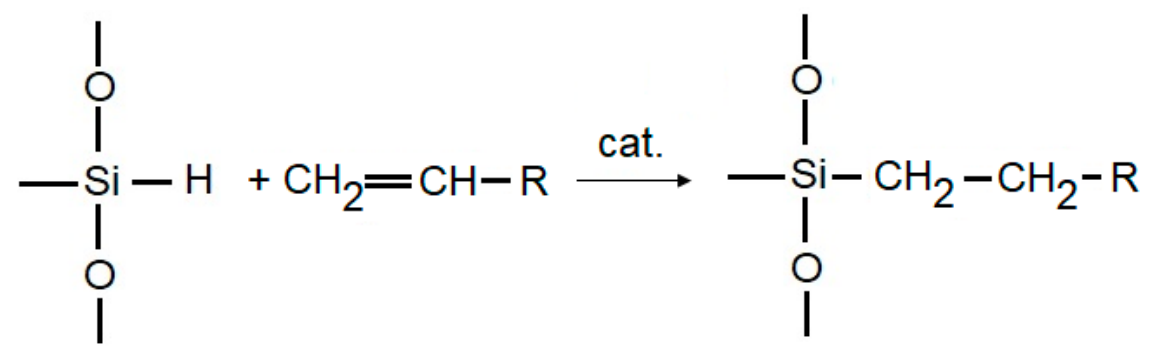

The reaction can utilize a wide range of organic compounds having a terminal double (alkenes) or triple (alkynes) bond and is easily done in a heterogeneous media (solid silica hydride in contact with the organic compound dissolved in an appropriate solvent). Therefore, a new surface can be tailored to the desired chromatographic properties by a selection of the appropriate alkene or alkyne. Optimum loading of the organic group is controlled by temperature, solvent, and catalyst (many types of metal complexes, such as hexachloroplatinic acid or free radical initiators, can be used).

An important difference can now be noted between stationary phases based on organosilanization of silica and hydrosilation of silica hydride. In both cases, due to steric considerations at best, no more than about $50 \%$ of the surface reactive groups (silanols on silica and $\mathrm{Si}-\mathrm{H}$ on silica hydride) can be modified. Thus the remaining groups are polar silanol groups on ordinary silica but mildly hydrophobic $\mathrm{Si}-\mathrm{H}$ moieties on silica hydride materials. A readily measurable difference between the two surfaces is the amount of water adsorbed. For stationary phases based on ordinary silica, there are between four and ten layers of water on the surface [6]. However, silica hydride has less than 0.5 of a monolayer of water on the surface due to its hydrophobic nature. This finding has a substantial impact on chromatographic differences that are found between stationary phases based on the two support materials that will be discussed later in this review.

In the development of any reaction protocol, reproducibility is a key issue. This is particularly true for the fabrication of stationary phases for HPLC because many types of analyses require retention times from run to run and from column to column to be extremely precise, usually with a standard deviation of a few percent or less. The protocol for the production of silica hydride-based stationary phases has been optimized since its inception and is now extremely reproducible as shown by the chromatograms in Figure 1. Similar chromatograms can be obtained for all of the different commercially available columns based on a silica hydride support: Bidentate C18, bidentate C8, Diamond Hydride, phenyl, cholesterol, undecanoic acid, diol, and amide. The term "bidentate" refers to the double attachment to the surface of the organic moiety due to the use of an alkyne in the hydrosilation reaction [7]. This synthetic approach then provides attachment to the surface by two stable silicon-carbon bonds. This feature is responsible for the long lifetime of these reversed-phase column materials. The versatility of the hydrosilation reaction has been demonstrated over the years by bonding a wide variety of alkenes and alkynes to the silica hydride surface [8-25]. Some examples of organic moieties attached to silica hydride and tested chromatographically as stationary phases include typical straight chain hydrophobic moieties for reversed-phase (1-octadecene, 1-octene, 4-phenyl-1-butene), large hydrophobic molecules (squalene), polar organic compounds (allyl glycidyl ether, 7-octene-1,2-diol), halogenated compounds (perfluoro-1-octene), charged moieties ( $N$-benzylcinchonidinium chloride), liquid crystals (cholesteryl-10-undecenoate) and potential chiral selectors ( $\beta$-cyclodextrin) [26]. To date only a few of the successful reactions developed have resulted in commercially available columns. In many cases, these materials have not been thoroughly tested so it may be possible that useful applications will be found for them in the future. 


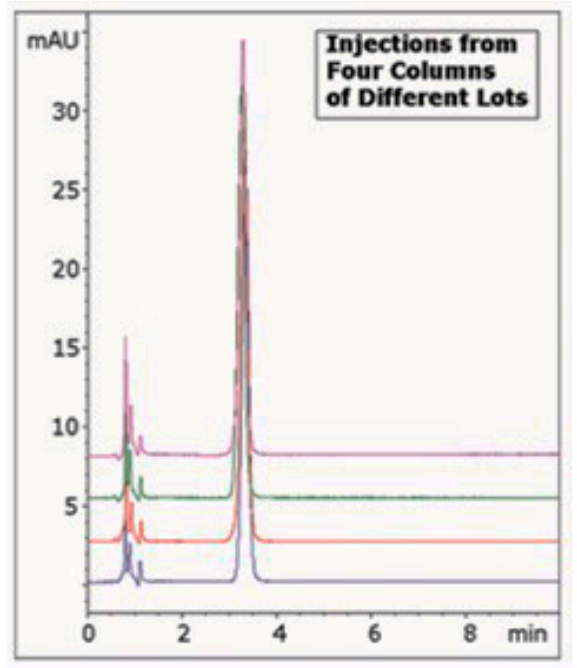

Figure 1. Overlay of chromatograms for the same analyte (diphenhydramine) taken on the same bonded phase (silica-C) of silica hydride columns made from four different synthetic batches. Mobile phase 50:50 acetonitrile/DI water $+5 \mathrm{mM}$ ammonium acetate. (http://kb.mtc-usa.com/article/AA-00918).

\section{Characterization of Silica Hydride Materials}

A crucial part of the fabrication of new materials for chromatographic stationary phases is characterization by physical and spectroscopic methods. There are several approaches that have been used to establish the success of the hydrosilation reaction on silica hydride and to verify the identity of the bonded organic moiety. One of the most frequently used spectroscopic techniques is diffuse reflectance infrared Fourier transform (DRIFT) spectroscopy [8,9,27-29]. This approach results in the infrared spectrum of the surface of the silica hydride particle. An example of such a spectrum is shown in Figure 2 for a butyl phenyl moiety bonded on a silica hydride surface. The essential features are as follows: just above $3000 \mathrm{~cm}^{-1}$ peaks are due to aromatic carbon-hydrogen stretch, just below $3000 \mathrm{~cm}^{-1}$ are peaks for aliphatic carbon-hydrogen stretch, and the large peak near $2250 \mathrm{~cm}^{-1}$ is the silicon-hydrogen stretch from the unreacted $\mathrm{Si}-\mathrm{H}$ groups on the surface. The remainder of the features in the spectrum are the result of silicon-oxygen matrix vibrational frequencies. Another useful spectroscopic technique is cross-polarization magic-angle spinning (CP-MAS) nuclear magnetic resonance (NMR) [30-32]. This method results in the NMR spectrum of the surface of solid particles such as typical chemically modified chromatographic supports. An example of a carbon-13 spectrum obtained for a silica hydride-based material with cholesterol bonded to the surface is shown in Figure 3. While the spectrum is complex, it shows the wide variety of aliphatic carbons, the two olefinic carbons and the carbonyl carbon that are part of the cholesterol moiety. Further corroboration of successful bonding via hydrosilation of silica hydride has also been obtained from the solid-state Si-29 CP-MAS NMR spectrum. These two characterization techniques provide sufficient documentation that the organic moiety used in the hydrosilation reaction was successfully bonded to the silica hydride support material. The extent of bonding is determined from elemental carbon analysis [33]. Using the molecular weight on the bonded moiety and the percent carbon, the surface coverage expressed in $\mu \mathrm{mol} / \mathrm{m}^{2} \mathrm{can}$ be calculated. As with organosilanization the extent of bonding (surface coverage) is controlled by the size of the organic moiety attached. Both ordinary silica and silica hydride have approximately $8 \mu \mathrm{mol} / \mathrm{m}^{2}$ of reactive groups (Si-OH for silica and Si-H for silica hydride) on the surface. For very small organic moieties as much as $4-5 \mu \mathrm{mol} / \mathrm{m}^{2}$ can be reacted, but for very large groups it can be as low as $2 \mu \mathrm{mol} / \mathrm{m}^{2}$ or less. The crucial difference between chemical modification of ordinary silica and silica hydride is that the unreacted groups are silanols on silica but $\mathrm{Si}-\mathrm{H}$ on silica hydride. Thus the underlying surface is very polar on silica but mildly hydrophobic on silica hydride. 


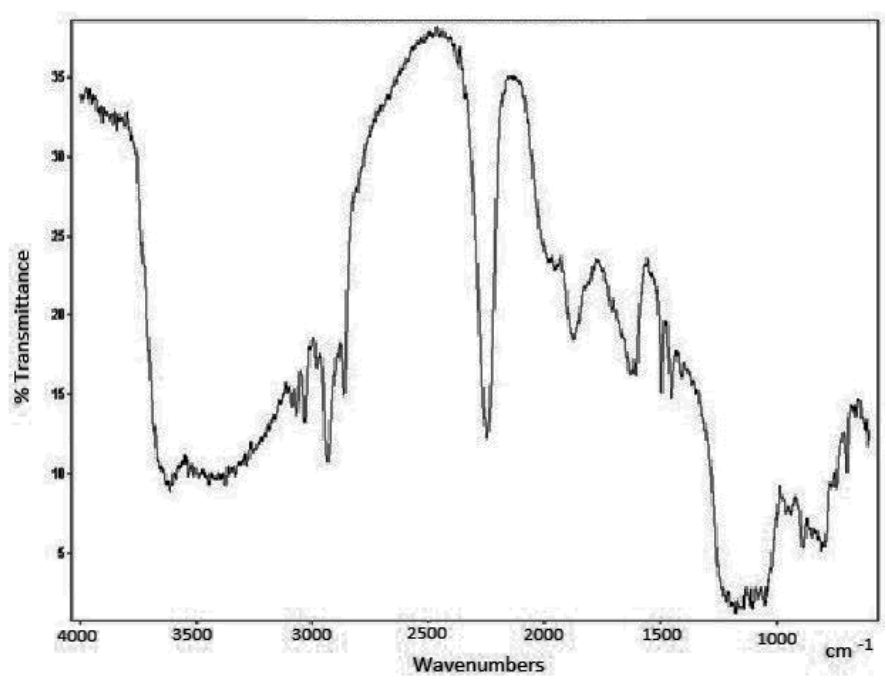

Figure 2. Diffuse reflectance infrared Fourier transform (DRIFT) spectrum of a butyl phenyl moiety bonded to a silica hydride surface.

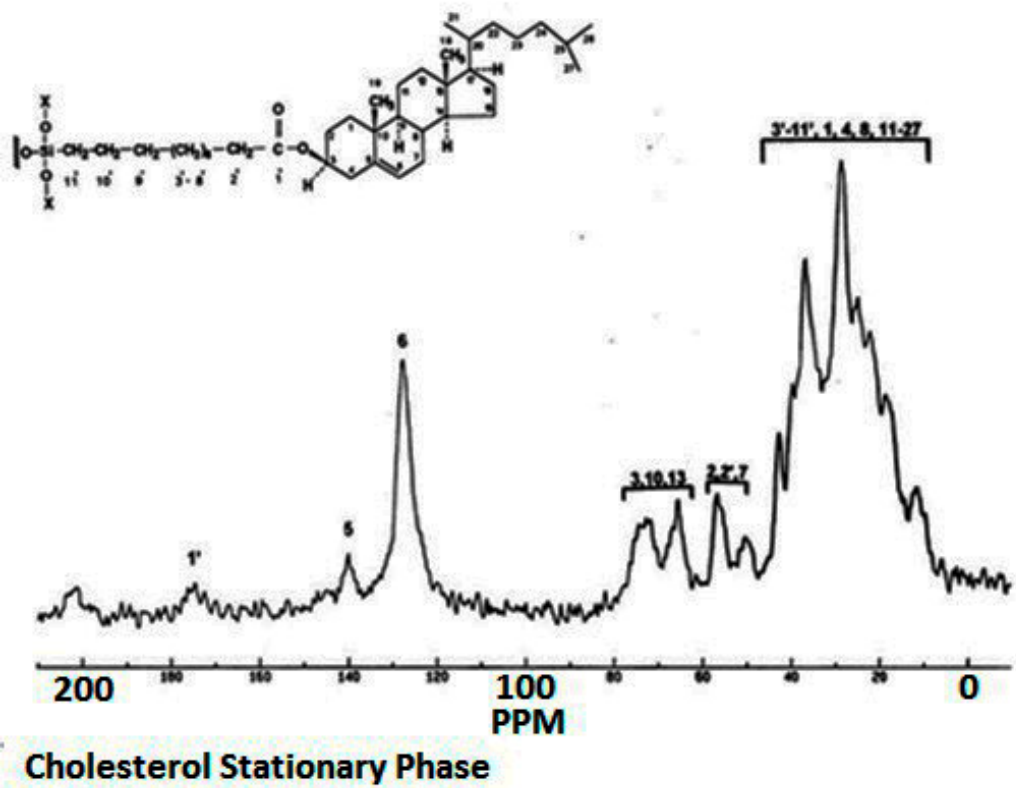

Figure 3. Carbon-13 cross-polarization magic-angle spinning (CP-MAS) nuclear magnetic resonance (NMR) spectrum of the silica hydride cholesterol stationary phase.

An important outcome of the differences in surface chemistry is the amount of water adsorbed by the two chromatographic supports. Table 1 lists a number of silica-based phases (top four) and silica hydride materials (bottom five indicated as Cogent) and their respective layers of adsorbed water [6]. As can be seen, the silica phases have multiple layers (up to 10) of water on the surface while the silica hydride phases have less than one-half of a monolayer on its surface. This property results in a unique chromatographic feature that is shown in Figure 4. All stationary phases based on silica hydride have dual retention mechanism as shown in a plot of retention time vs. mobile phase composition [34]. At high water composition, reversed phase retention for hydrophobic compounds is obtained while at high organic composition normal phase retention is observed for hydrophilic compounds. Therefore, both modes can be tested for a particular sample and the best mode can be selected for developing an analytical format. Except for mixed-mode phases [35-38], most common stationary phases offer only one mode for doing separations, either reversed-phase or normal phase. 
Table 1. Number of water layers on chromatographic stationary phases.

\begin{tabular}{cc}
\hline Column & $\mathbf{N}_{\mathbf{w}}$ \\
\hline ZIC HILIC & 6.11 \\
ZIC cHILIC & 9.48 \\
Luna HILIC & 4.72 \\
Triart DIOL & 3.06 \\
Cogent Silica C & 0.45 \\
Cogent Diamond hydride & 0.43 \\
Cogent UDC Cholesterol & 0.32 \\
Cogent Bidentate C18 & 0.28 \\
Cogent Phenyl hydride & 0.23 \\
\hline
\end{tabular}

Data taken from [34].

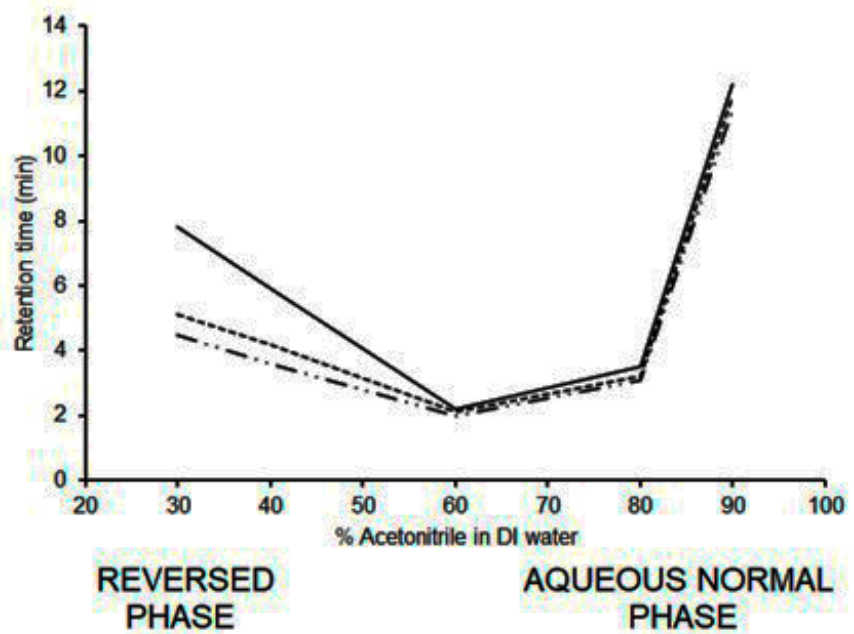

Figure 4. Retention map of typical silica hydride-based stationary phases illustrating the dual retention properties of these materials.

\section{Separation Mechanisms on Silica Hydride}

An interesting challenge with respect to silica hydride-based stationary phases is determining the mechanism that allows these materials to operate in both the reversed-phase and normal-phase modes. The reversed-phase capability is easily explained by ordinary hydrophobic interactions. If the bonded moiety has any hydrophobic properties (and most have at least a minimal amount) plus the mildly hydrophobic nature of the hydride surface, then at least some reversed-phase capabilities are to be expected. However, a more challenging problem is to determine what is responsible for the normal-phase retention properties with respect to polar compounds. The first clue to solving this problem was obtained by measuring the zeta potential of various silica hydride-based stationary phases [39]. The zeta potential provides a measurement of the surface charge on the stationary phase material. In the case of all silica hydride-based stationary phases, it was determined that each had a substantial negative charge on the surface when it is in contact with a high organic content liquid. Such a result was not expected because silica hydride is fabricated by removing more than $95 \%$ of silanol groups with the resulting surface being hydrophobic as measured chromatographically under reversed-phase conditions. However, recent investigations [39] have determined that the negative charge on the silica hydride surface is due to hydroxyls from the auto-dissociation of water. This result is similar to those obtained for oil droplets in mixed organo-aqueous solvents [40]. Since the silica hydride particle is hydrophobic in a way that is similar to an oil droplet, the assumption that two behave similarly in comparable environments is a good one. This model accounts for the fact that there is only a small amount of water on the surface, but some of it auto dissociates to give $\mathrm{OH}^{-}$where the 
negative charge is measured, and the $\mathrm{H}^{+}$is below the surface and does not affect the chromatographic properties. Thus, retention in the normal phase mode is due to either charge attraction for positive or positively polarized analytes and charge displacement for negative or negatively polarized analytes.

A particularly good example of the unusual properties of the silica hydride-based phases is demonstrated by chromatographic results obtained for the highly polar chemotherapy agent and immunosuppressant methotrexate. A representative chromatogram for this compound is shown in Figure 5. The unique feature of the analysis shown is that this hydrophilic compound is strongly retained on a silica hydride material modified with an octadecyl (C18) moiety. This result would not be possible on an ordinary silica C18 stationary phase that only retains compounds in the reversed-phase mode. Using a mobile phase with a high aqueous content would produce some retention based on the weakly hydrophobic character of the molecule but would be much weaker than the strong interaction of methotrexate with the silica hydride phase in the aqueous normal phase mode. While characterizing and identifying the unique properties of a new separation material is important, the final step and ultimately most relevant is to determine how such a stationary phase can provide analytical capabilities that are useful in practical applications. The U-shaped retention behavior shown in Figure 4 can be used advantageously for complex samples particularly when the two modes overlap. Thus, there is the ability to retain both hydrophobic and hydrophilic compounds in a single analysis. A simple example is shown in Figure 6 where two compounds, one polar (phenylglycine) and one nonpolar (sertraline), both exhibit significant retention under the same analytical conditions. The column used in this analysis was the Diamond Hydride, a silica hydride stationary phase with only a minimal amount of an organic moiety ( $2 \%$ carbon) on the surface. Retention of both of these diverse compounds illustrates the hydrophobic properties of the silica hydride group and the presence of the hydroxyl ions for polar retention. Another more challenging application is illustrated in Figure 7. Here a mixture of peptides ranging from very hydrophobic to very hydrophilic is separated in a single run on the same Diamond Hydride column [41]. Such a result is not possible on other columns and in fact, most proteomic analyses are done using a two-dimensional approach. One run was done on a hydrophobic phase such as $\mathrm{C} 18$ and the second run was done on a more polar medium such as ion-exchange. Because of the versatility of the silica hydride columns, many analyses can be attempted by three possible approaches: reversed-phase, aqueous normal-phase, or a method that combines some of both modes so that both hydrophobic and hydrophilic compounds can be retained simultaneously.

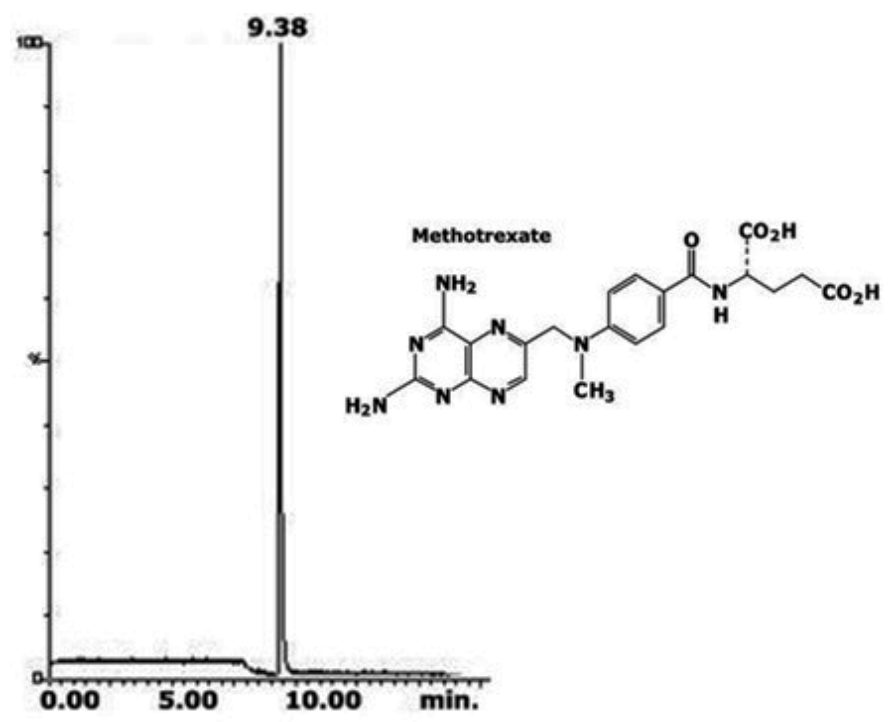

Figure 5. Analysis of methotrexate using a silica hydride $\mathrm{C} 18$ stationary phase in the aqueous normal phase mode. Mobile phase: A, DI $\mathrm{H}_{2} \mathrm{O} / 0.5 \%$ formic acid and B, acetonitrile. Gradient: 0-1 min 90\% B, 1-5 min to $20 \%$ B, 5-10 min 20\% B. (http://kb.mtc-usa.com/article/AA-00784/0/). 


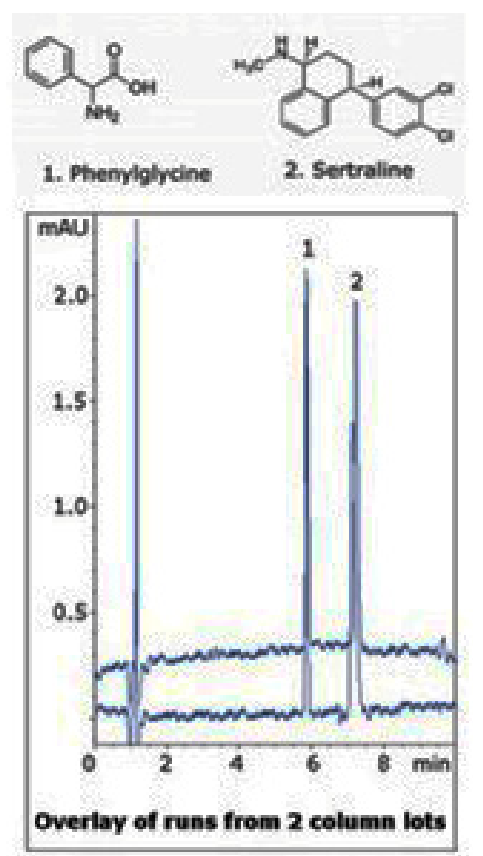

Figure 6. Separation of a polar and nonpolar compound in a single run on a silica hydride-based (Diamond Hydride) HPLC column. Mobile phase: A, DI water $+0.1 \%$ formic acid and B acetonitrile + 0.1\% formic acid. Gradient: 0-1 $\mathrm{min} 95 \%$ B, 1-6 $\mathrm{min}$ to 50\% B. (http://kb.mtc-usa.com/article/AA-01460/0/).

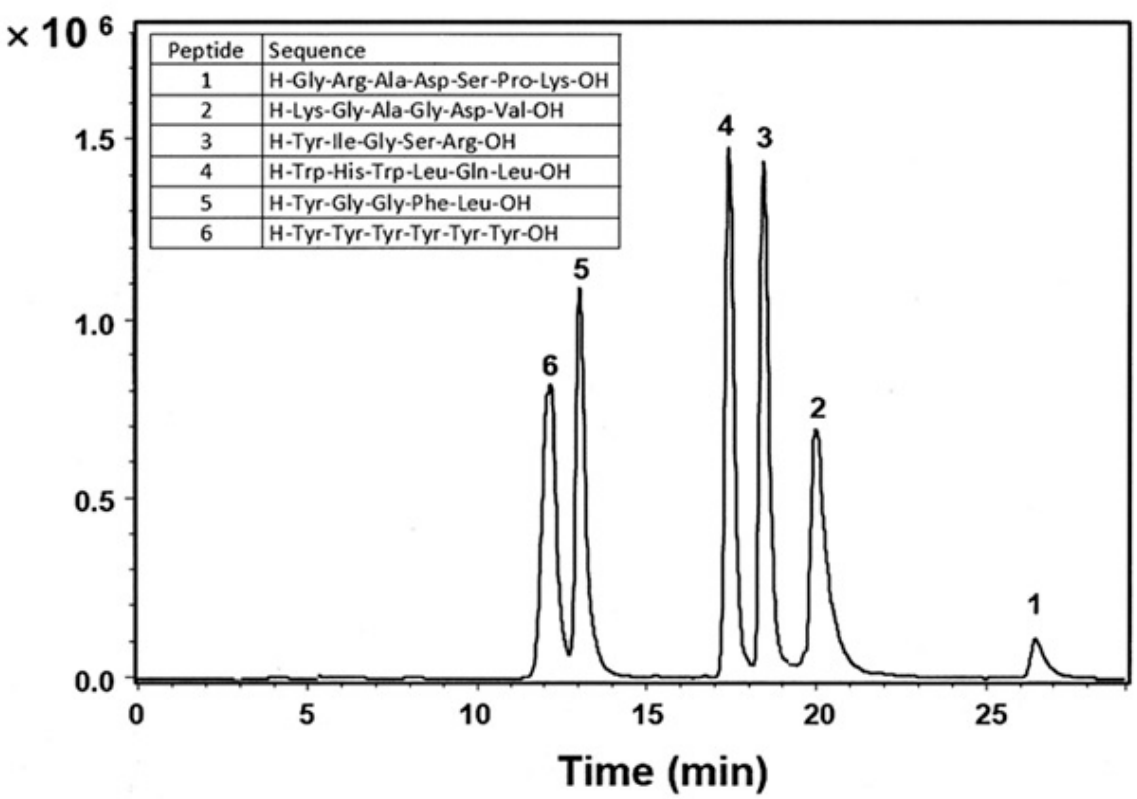

Figure 7. Separation of a mixture containing both hydrophobic and hydrophilic peptides in a single run on the Diamond Hydride column under aqueous normal phase conditions. Adapted from data in Reference [41].

\section{Applications of Silica Hydride HPLC Columns}

Because of the extensive work already done on Type $C$ columns, such as some of those found in the literature [42-65], a summary of all possible applications is not possible. A few examples will be presented to illustrate some of the capabilities of these unique stationary phases.

An area of growing importance is that of food analysis. Methods have been developed for food quality, purity, and possible health benefits. The determination of polyphenols is an active area of investigation for many food products. Polyphenols fall into the general category of anti-oxidants, 
which have been shown to have the potential for preventing and curing cancer as well as other diseases. Figure 8 shows the analysis of two polyphenols, rutin, and quercetin, that have been identified as having potential health and medicinal benefits [66]. Rutin is a citrus flavonoid glycoside found in many plants including buckwheat and asparagus. Rutin is also found in citrus fruits such as orange, grapefruit, lemon, and lime, apple, berries such as mulberry, clingstone' peaches, and green tea infusions. Quercetin is found a wide variety of food sources including red kidney beans, cilantro, dill, red onion, kale, cranberry, sweet potato, blueberry, broccoli, and tea. Both of these compounds are analyzed on the Diamond Hydride column in the aqueous normal-phase mode because of the multiple polar hydroxy groups in their structures.

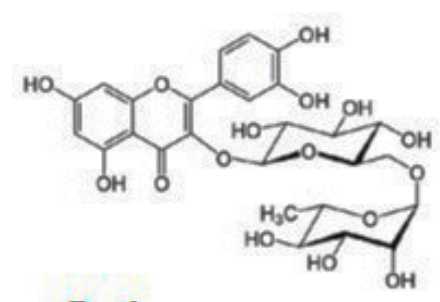

Rutin<smiles>O=C1CC(O)CC2(Oc3ccc(O)c(O)c3)C(=O)C(O)C(O)C12</smiles>

Quercetin

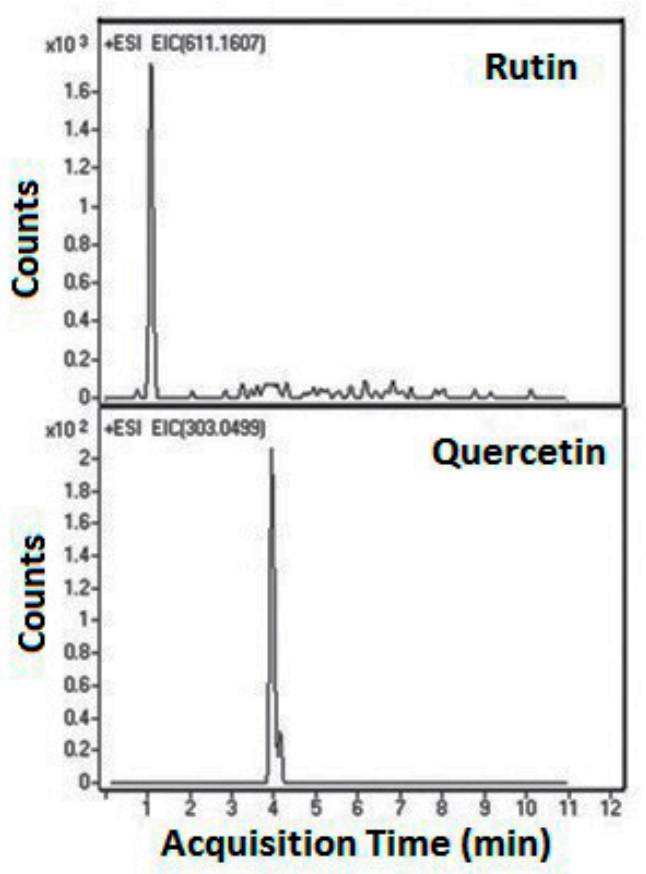

Figure 8. Analysis of the polyphenols rutin and quercetin on the Diamond Hydride column in the aqueous normal-phase mode. Adapted from Reference [66].

The analysis of metabolites is an important aspect of pharmacokinetic studies, understanding various physiological and biological systems, clinical tests and food production. These determinations are almost always made in complex matrices with hundreds and in some cases even thousands of compounds in many samples. Figure 9 shows the analysis of some crucial components in the uric acid cycle along with the internal standard tyrosine [67]. This metabolite is crucial in a number of physiological functions, and the concentrations of these compounds can potentially be used in disease diagnosis. As shown in the two extracted ion chromatograms, the analysis of these compounds in a real physiological sample parallels the data obtained with standards. Thus, matrix effects for this silica hydride-based (Type C) column (Cogent Diol) are minimal for this analysis in the aqueous normal-phase mode. 


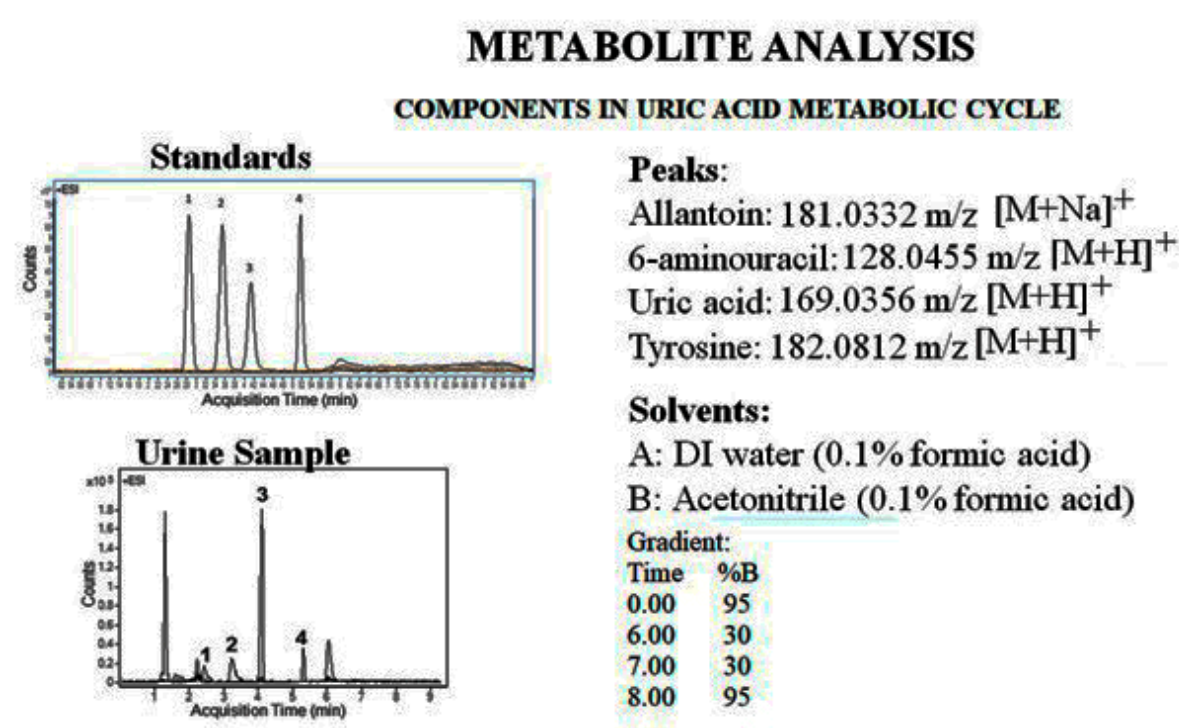

Figure 9. Analysis of components in the uric acid on the Cogent Diol stationary phase in the aqueous normal-phase mode. Adapted from Reference [67].

Another area of growing importance in chemical analysis are investigations and method development related to the forensic field. Of the various aspects of forensic analysis, methods related to illicit drugs are among the most important. Figure 10 is an LCMS analysis of several of the street drugs classified as "bath salts". The chemical category of these compounds is cathinones, and the basic structure is also shown in Figure 10. In this analysis, the column used is the phenyl phase operating in the reversed-phase mode [68]. The phenyl column is used because as can be seen the cathinone moiety has an aromatic ring as a primary constituent. Thus pi-pi interactions between the analyte and the stationary phase can offer a means for discriminating among the various analytes. The chromatogram shown in Figure 10 illustrates the excellent selectivity and efficiency that silica hydride-based stationary phases offer in the reversed-phase mode. The unique surface composed of Si-H moieties in contrast to ordinary silica phases where residual silanols are present results in enhanced chromatographic capabilities in both reversed-phase and normal-phase applications.
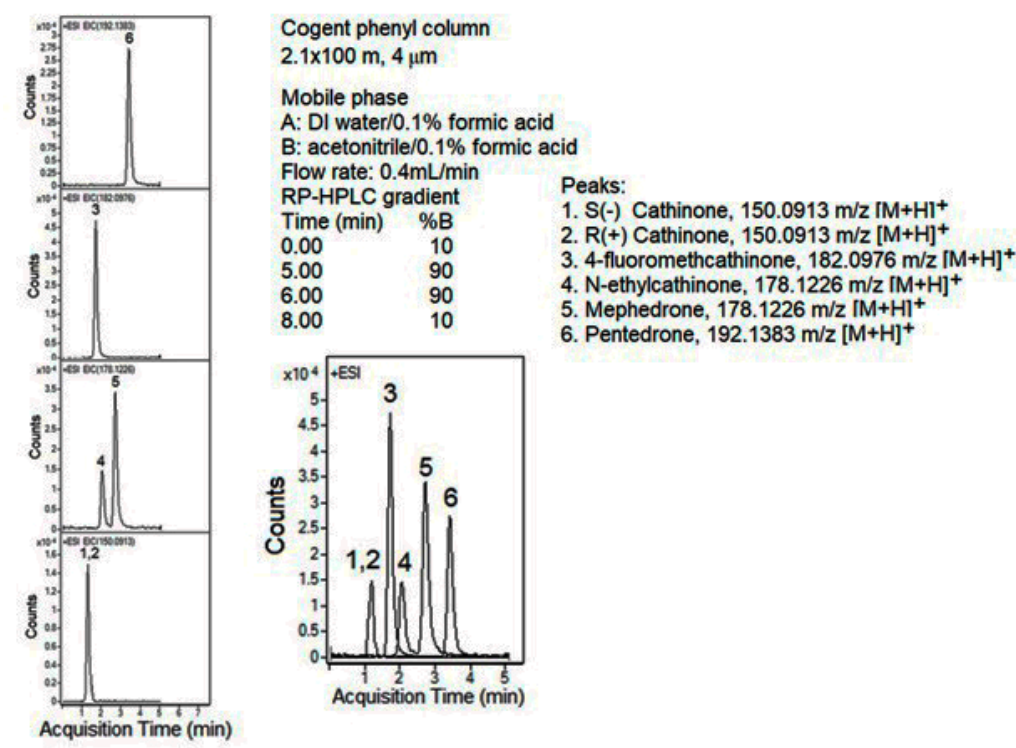

Figure 10. Separation of a mixture of the illicit street drugs "bath salts" on the silica hydride-based phenyl column in the reversed-phase mode. Data taken from Reference [68]. 
The analysis of pharmaceuticals, both name-brand and generic, has been an active area of investigation using silica hydride columns in both the reversed-phase and aqueous normal phase modes. Because a significant number of pharmaceuticals are polar compounds, the aqueous normal phase mode using the Diamond Hydride column provides an excellent tool in order to develop reliable analytical protocols for these types of drugs. Figure 11 illustrates the typical results that are obtained with a polar pharmaceutical compound (atenolol) on the Diamond Hydride in the aqueous normal phase mode [69]. Both significant retention and excellent peak shape can be achieved for this hydrophilic compound. Also demonstrated in this figure is the batch-to-batch reproducibility of fabricating this stationary phase as the analyte peak from the two lots are nearly superimposable in the chromatograms. These unique capabilities have made silica hydride-based stationary phases a superior approach to hydrophobic interaction liquid chromatography (HILIC) when developing analytical protocols for polar compounds. In addition, the hydride surface is less adsorptive that ordinary silica. This property results in less contamination of the stationary phase when complex biological matrices are analyzed and when some deterioration of performance does occur it is much easier to remove the contaminants with column washing than with ordinary silica-based materials.

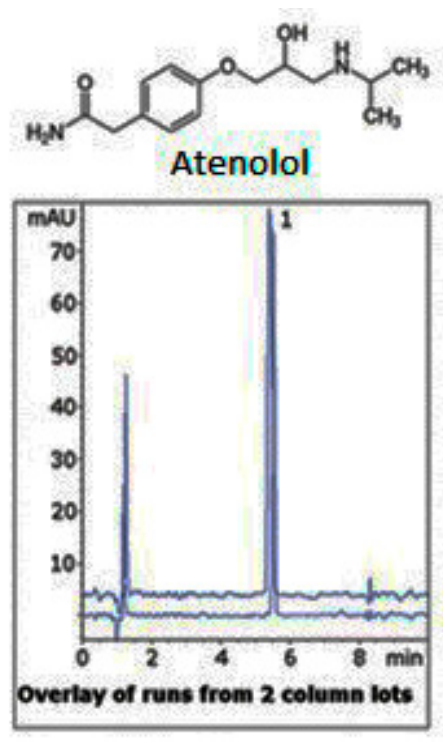

Figure 11. Analysis of the pharmaceutical compound atenolol on the Diamond Hydride column using the aqueous normal phase mode. Adapted from Reference [69].

A more exhaustive compilation of applications can be found in the Knowledge Base section of the Microsolv Technology web site (http://kb.mtc-usa.com/).

\section{Conclusions}

The use of silica hydride-based stationary phases (Type-C silica) has grown steadily over the last 15 years based on the unique properties these materials possess. The dominant feature of silica hydride with respect to ordinary silica is the presence of $\mathrm{Si}-\mathrm{H}$ groups on the surface in contrast to silanols. This results in a very thin water layer $(<0.5$ monolayer $)$ on silica hydride while on ordinary silica there is a more substantial amount of water (3-10 monolayers). This difference gives silica hydride properties that can be exploited for solving difficult analytical problems. In particular, for the analysis of polar compounds using the aqueous normal-phase mode in comparison to HILIC, there is excellent run-to-run, day-to-day and batch-to-batch reproducibility, good peak shape and fast re-equilibration between runs particularly for gradients and easy clean-up when analyzing biological matrices. All of the silica hydride phases can be used in either the reversed-phase or aqueous normal-phase modes. The extent of retention in each of the modes is determined by the organic moiety bonded to the surface. With little or no modification, or with a very small or polar moiety bonded, the aqueous 
normal-phase mode is dominant. With more hydrophobic bonded groups, the reversed-phase mode is more prevalent. Differences in selectivity, when compared to reversed-phase materials on ordinary silica, are also observed. Overall, silica hydride-based separation materials offer a new approach to solving challenging analytical problems in complex samples and matrices.

Funding: The authors gratefully acknowledge the funding provided by the following organizations over the last 20 years that have supported the research involved in developing silica hydride stationary phases: National Science Foundation; National Institutes of Health; W.M. Keck Foundation; Agilent Foundation; and the Camille and Henry Dreyfus Foundation.

Acknowledgments: The authors thank the following organizations and individuals for collaborations during the development of silica hydride separation materials: Microsolv Technology Corporation; Agilent Technologies; Milton Hearn and Reinhard Boysen of Monash University, Melbourne, Australia.

Conflicts of Interest: Both authors provide consulting services to Microsolv Technology Corp.

\section{References}

1. Unger, K.K. Porous Silica; Elsevier Science: Amsterdam, The Netherlands, 1979; ISBN 97800808166.

2. Pesek, J.J.; Swedberg, S.A. Allyl bonded stationary Phase as a possible intermediate in the synthesis of novel high-performance liquid chromatographic phases. J. Chromatogr. 1986, 361, 83-109. [CrossRef]

3. Sandoval, J.E.; Pesek, J.J. Synthesis and characterization of a hydride modified porous silica material as an intermediate in the preparation of chemically bonded chromatographic stationary phases. Anal. Chem. 1989, 61, 2067-2075. [CrossRef]

4. Sandoval, J.E.; Pesek, J.J. Hydrolytically stable bonded chromatographic phases prepared through hydrosilation of olefins on a hydride-modified silica intermediate. Anal. Chem. 1991, 63, 2634-2641. [CrossRef]

5. Chu, C.H.; Jonsson, E.; Auvinen, M.; Pesek, J.J.; Sandoval, J.E. A new approach for the preparation of a hydride-modified substrate used as an intermediate in the synthesis of surface-bonded materials. Anal. Chem. 1993, 65, 808-816. [CrossRef]

6. Soukup, J.; Jandera, P. Adsorption of water from aqueous acetonitrile on silica-based stationary phases in aqueous normal-phase liquid chromatography. J. Chromatogr. A 2014, 1374, 102-111. [CrossRef] [PubMed]

7. Pesek, J.J.; Matyska, M.T.; Oliva, M.; Evanchic, M. Synthesis and characterization of bonded phases made via hydrosilation of alkynes on silica hydride surfaces. J. Chromatogr. A 1998, 818, 145-154. [CrossRef]

8. Pesek, J.J.; Matyska, M.T.; Soczewinski, E.; Christensen, P. Spectroscopic studies of butylphenyl, mono-ol and perfluorinated bonded phases. Chromatographia 1994, 39, 520-528. [CrossRef]

9. Pesek, J.J.; Matyska, M.T. Synthesis and spectroscopic characterization of a true diol bonded phase. J. Chromatogr. 1994, 687, 33-44. [CrossRef]

10. Pesek, J.J.; Matyska, M.T.; Hemphala, H. HPLC evaluation of mono-ol, butylphenyl, and perfluorinated columns prepared via olefin hydrosilation on a silica hydride intermediate. Chromatographia 1996, 43, 10-16. [CrossRef]

11. Pesek, J.J.; Matyska, M.T.; Williamsen, E.J.; Evanchic, M.; Hazari, V.; Konjuh, K.; Takhar, S.; Tranchina, R. The synthesis and characterization of alkyl bonded phases from a silica hydride intermediate via hydrosilation with free radical initiation. J. Chromatogr. A 1997, 786, 219-228. [CrossRef]

12. Pesek, J.J.; Matyska, M.T.; Williamsen, E.; Tam, R.; Wang, Z. Synthesis and characterization of liquid crystal type stationary phases on a silica hydride surface. J. Liq. Chromatogr. Rel. Technol. 1998, 21, 2747-2762. [CrossRef]

13. Pesek, J.J.; Matyska, M.T.; Takhar, S. Synthesis and characterization of long chain alkyl stationary phases on a silica hydride surface. Chromatographia 1998, 48, 631-636. [CrossRef]

14. Pesek, J.J.; Matyska, M.T.; Muley, S. Synthesis and characterization of a new type of chemically bonded liquid crystal stationary phase for HPLC. Chromatographia 2000, 52, 439-444. [CrossRef]

15. Pesek, J.; Matyska, M.T.; Fu, P.F. Evaluation of the silanization/hydrosilation process for the synthesis of chiral stationary phases. Chromatographia 2001, 53, 635-640. [CrossRef]

16. Matyska, M.T.; Pesek, J.J.; Grandhi, V. Charge transfer-like stationary phase for HPLC prepared via hydrosilation on silica hydride. J. Sep. Sci. 2002, 25, 741-748. [CrossRef] 
17. Pesek, J.J.; Matyska, M.T.; James, S. Synthesis and characterization of a C8 stationary phase bonded with 2-acrylamido-2-methyl-1-propanesulfonic acid for HPLC. J. Liq. Chromatogr. Rel. Technol. 2002, 25, $2749-2765$. [CrossRef]

18. Pesek, J.J.; Matyska, M.T.; Dawson, G.B.; Wilsdorf, A.; Marc, P.; Padki, M. The cholesterol bonded phase as a separation medium in high performance liquid chromatography. Evaluation of properties and applications. J. Chromatogr. A 2003, 986, 253-262. [CrossRef]

19. Matyska, M.T.; Pesek, J.J.; Tong, S.; Sandoval, J.E. Adamantyl-modified silica via olefin hydrosilation on a hydride Intermediate. J. Liq. Chromatogr. Rel. Technol. 2003, 26, 1169-1195. [CrossRef]

20. Matyska, M.T.; Pesek, J.J.; Pan, X. Synthesis and evaluation of a C8 phase on a silica hydride surface by hydrosilation of 1-octyne. J. Chromatogr. A 2003, 992, 57-65.

21. Matyska, M.T.; Pesek, J.J.; Suryadevara, R. Synthesis and characterization of amino-based columns for HPLC made by silanization/ hydrosilation. J. Liq. Chromatogr. Rel. Technol. 2005, 28, 2111-2139. [CrossRef]

22. Pesek, J.J.; Matyska, M.T.; Prabhakaran, S. Synthesis and characterization of chemically bonded stationary phases on hydride surfaces by hydrosilation of alkynes and dienes. J. Sep. Sci. 2005, 28, 2437-2443. [CrossRef]

23. Pesek, J.J.; Matyska, M.T.; Prajapati, K. Synthesis and evaluation of silica hydride-based fluorinated stationary phases. J. Sep. Sci. 2010, 33, 2908-2916. [CrossRef]

24. Pesek, J.J.; Matyska, M.T.; Lee, P. Synthesis of a preparative C30 stationary phase on a silica hydride surface and its application to carotenoid separation. J. Liq. Chromatogr. Rel. Technol. 2011, 34, 231-240. [CrossRef]

25. Pesek, J.J.; Matyska, M.T.; Salehi, N. Evaluation of stationary phases made by hydrosilation of alkynes on silica hydride. Curr. Chromatogr. 2015, 2, 41-47. [CrossRef]

26. Pesek, J.J.; Matyska, M.T. Silica hydride surfaces: Versatile separation media for chromatographic and electrophoretic analyses. J. Liq. Chromatogr. Rel. Technol. 2006, 29, 1105-1124. [CrossRef]

27. Pesek, J.J.; Williamsen, E. Spectroscopic characterization of chemically modified oxide surfaces. Trends Appl. Spectrosc. 1993, 1, 41-50.

28. Pesek, J.J.; Matyska, M.T. Methods for the modification and characterization of oxide surfaces. J. Interface Sci. 1997, 5, 103-117. [CrossRef]

29. Pesek, J.J.; Matyska, M.T. Spectroscopic characterization of chemically modified oxide surfaces. In Adsorption and Its Application in Industry and Environmental Protection; Dabrowski, A., Ed.; Elsevier: Amsterdam, The Netherlands, 1999; Volume 1, pp. 117-142.

30. Pesek, J.J.; Matyska, M.T.; Williamsen, E.J.; Tam, R. Variable-temperature solid-state NMR studies of bonded liquid crystal stationary phases for HPLC. Chromatographia 1995, 41, 301-310. [CrossRef]

31. Freibolin, V.; Bayer, M.P.; Matyska, M.T.; Pesek, J.J.; Albert, K. ${ }^{1}$ H HR/MAS NMR in the suspended state: Molecular recognition processes in liquid chromatography between steroids and a silica hydride-based cholesterol phase. J. Sep. Sci. 2009, 32, 1722-1728. [CrossRef]

32. Yeman, H.; Friebolin, V.; Steinhauser, L.; Matyska, M.T.; Pesek, J.J.; Albert, K. Time dependant column performance of cholesterol based stationary phases for HPLC by LC characterization and solid state NMR spectroscopy. J. Sep. Sci. 2012, 35, 1582-1588. [CrossRef]

33. Berendsen, G.E.; De Galan, L. Preparation and chromatographic properties of some chemically bonded phases for reversed-phase liquid chromatography. J. Liq. Chromatogr. 1978, 1, 561-568. [CrossRef]

34. Pesek, J.J.; Matyska, M.T. How to retain polar and nonpolar Compounds on the same HPLC Column with an isocratic mobile phase. LC/GC 2006, 24, 296-303.

35. Zhang, L.; Dai, Q.; Qiao, X.; Yu, C.; Qin, X.; Yan, H. Mixed-mode chromatographic stationary phases: Recent advancements and its applications for high-performance liquid Chromatography. TrAC Trends Anal. Chem. 2016, 82, 143-163. [CrossRef]

36. Zhang, K.; Xiaodong, L. Mixed-mode chromatography in pharmaceutical and biopharmaceutical applications. J. Pharm. Biomed. Anal. 2016, 128, 73-88. [CrossRef]

37. Wang, L.; Wei, W.; Zhining, X.; Xu, J.; Zeng, Z.X. Recent advance in materials form stationary phases of mixed-mode high-performance liquid chromatography. TrAC Trends Anal. Chem. 2016, 80, 495-506. [CrossRef]

38. Sykora, E.; Rezanak, P.; Zaruba, K.; Kral, V. Recent advances in mixed-mode chromatographic stationary phases. J. Sep. Sci. 2019, 42, 89-129. [CrossRef] 
39. Kulsing, C.; Yang, Y.; Munera, C.; Tse, C.; Matyska, M.T.; Pesek, J.J.; Boysen, R.I.; Hearn, M.T.W. Correlation between zeta potential and analyte retention using ionic interaction descriptors for silica hydride-based stationary phases. Anal. Chim. Acta 2014, 817, 48-60. [CrossRef]

40. Kulsing, C.; Nolvachi, Y.; Marriott, P.; Boysen, R.; Matyska, M.; Pesek, J.; Hearn, M. Insights into the origin of the separation selectivity with silica hydride adsorbents. J. Phys. Chem. Part B 2015, 119, 3063-3069. [CrossRef]

41. Boysen, R.I.; Yang, Y.; Chowdhury, J.; Matyska, M.T.; Pesek, J.J.; Hearn, M.T.W. Simultaneous separation of hydrophobic and hydrophilic peptides with a silica hydride stationary phase using aqueous normal phase conditions. J. Chromatogr. A 2011, 1218, 8021-8026. [CrossRef]

42. Nguyen, T.; Felker, P.; Hurst, J.W.; Ly, C.; Jarman, S.; Diep, D.; Pham, C.; Pesek, J.J.; Matyska, M.T.; Young, J.E.; et al. LC-MS characterization of phenolics in mesquite flour. LCGC N. Am. 2016, 34, 28-31.

43. Pesek, J.; Matyska, M.; Hoffman, J.F.; Madruga, N.; Crizel, R.I.; Elias, M.C.; Vanier, N.L.; Chaves, F.C. Analysis of mycotoxins in grains using silica hydride-based stationary phases. J. Sep. Sci. 2017, 40, 1953-1959. [CrossRef]

44. Young, J.E.; Pan, Z.; Teh, H.E.; Menon, V.; Modegerer, B.; Pesek, J.J.; Matyska, M.T.; Takeoka, G. Phenolic composition of phenolic extracts using an LCMS approach with silica hydride columns. J. Sep. Sci. 2017, 40, 1449-1456. [CrossRef]

45. Pesek, J.J.; Matyska, M.T.; Sieng, M.; Doan, L. Analysis of capsaicinoids in hot sauces using a silica hydride-based stationary phase. Curr. Chromatogr. 2016, 3, 12-16. [CrossRef]

46. Young, J.E.; Lim, M.V.; Topete, J.; Hang, H.; Gahol, M.; Pesek, J.J.; Matyska, M.T. Improved sensitivity and specificity of trans-resveratrol in red wine analysis with HPLC-UV and LC-MS. LCGC N. Am. 2016, 34, 205-213.

47. Dang, A.; Sieng, M.; Pesek, J.J.; Matyska, M.T. Determination of bisphenol A in receipts and carbon paper by HPLC-UV. J. Liq. Chromatogr. Rel. Technol. 2015, 38, 438-442. [CrossRef]

48. D'Orazio, G.; Rocco, A.; Fanali, S. Fast liquid chromatography using columns of different internal diameters packed with sub-2 $\mu \mathrm{m}$ silica particles. J. Chromatogr. A 2012, 1228, 213-220. [CrossRef]

49. Pesek, J.J.; Matyska, M.T.; Fischer, S.M.; Sana, T.R. Analysis of hydrophilic metabolites by high-performance liquid chromatography-mass spectrometry using a silica hydride-based stationary phase. J. Chromatogr. A 2008, 1204, 48-55. [CrossRef]

50. Chalkraft, K.R.; McCarry, B.E. Tandem LC columns for the simultaneous retention of polar and nonpolar molecules in comprehensive metabolomic analysis. J. Sep. Sci. 2013, 36, 3478-3485. [CrossRef]

51. Gouzy, A.; Larrouy-Maumus, G. Mycobacterium tuberculosis exploits asparagine to assimilate nitrogen and resist acid stress during infection. PLoS Pathogens 2014, 10, e1003928. [CrossRef]

52. Jenkins, S.; Fischer, S.M.; Chen, L.; Sana, T.R. Global LC/MS metabolomics profiling of calcium stressed and immunosuppressant treated Saccharomyces cerevisiae. Metabolites 2013, 3, 1102-1117. [CrossRef]

53. Putluria, N.; Shojaiegn, A.; Vasu, V.T. Metabolic profiling reveals potential markers and bioprocesses altered in bladder cancer progression. Cancer Res. 2011, 15, 7376-7386. [CrossRef]

54. Hellmuth, C.; Koletzko, B.; Peissner, W. Aqueous normal phase cysteine and methionine by liquid chromatography-tandem mass spectrometry. J. Chromatogr. B 2011, 879, 83-89. [CrossRef]

55. Gouzy, A.; Larrouy-Maumus, G. Mycobacterium tuberculosis nitrogen assimilation and host colonization require aspartate. Nat. Chem. Biol. 2013, 9, 674-676. [CrossRef]

56. Sana, T.R.; Gordon, D.B.; Fischer, S.M.; Tichy, S.E.; Kitagawa, N.; Cai, C.; Gosnell, W.L.; Chang, S.P. Global mass spectrometry based metabolomics profiling of erythrocytes infected with Plasmodium falciparum. PLoS ONE 2013, 8, e60840. [CrossRef]

57. Eoh, H.; Rhee, K.Y. Multifunctional essentiality of succinate metabolism in adaptation to hypoxia in mycobacterium tuberculosis. Proc. Natl. Acad. Sci. USA 2013, 110, 6454-6459. [CrossRef]

58. Chakraborty, S.; Gruber, T.; Barry, C.E., III; Boshoff, H.I.; Rhee, K.Y. Para-aminosalicylic acid acts as an alternate substrate of folate metabolism in mycobacterium tuberculosis. Science 2013, 339, 88-91. [CrossRef]

59. Cifkova, E.; Hajek, R.; Lisa, M.; Holcapek, M. Hydrophilic liquid interaction chromatography-mass spectrometry of (lyso)phosphaditic acids, (lyso)phophaditylserines and other lipid classes. J. Chromatogr. A 2016, 1450, 65-73. [CrossRef] 
60. Webster, G.K.; Elliott, A.; Dahan, A.; Miller, J.M. Analysis of PEG 400 in perfusate samples aqueous normal phase (ANP) chromatography with evaporative light scattering detection. Anal. Methods 2011, 1218, 742-744. [CrossRef]

61. Dang, A.; Matyska, M.T.; Pesek, J.J. The use of aqueous normal phase chromatography as an analytical tool for food analysis. Determination of histamine as a model system. Food Chem. 2013, 141, 4226-4230. [CrossRef]

62. Young, J.; Matyska, M.T.; Pesek, J.J. Robust HPLC-refractive index analysis of simple sugars in beverages using silica hydride columns. Curr. Nutr. Food Sci. 2016, 12, 125-131. [CrossRef]

63. Le, R.; Young, J.E.; Pesek, J.J.; Matyska, M.T. Separation of 1,3-Dimethylamine and other polar compounds in dietary supplement formulations using aqueous normal phase chromatography with mass spectrometry. J. Sep. Sci. 2013, 36, 2578-2583. [CrossRef]

64. Gea, J.; Liu, F.; Holmes, E.H.; Ostrander, G.K.; Li, Q.X. Aqueous normal phase liquid chromatography coupled with tandem time-of-flight quadrupole mass spectrometry for determination of zanamivir in human serum. J. Chromatogr. B 2012, 906, 58-62. [CrossRef]

65. Naffa, R.; Holmes, G.; Meekyung, A.; Harding, D.; Norris, G. Liquid chromatography electrospray ionization mass spectrometry for the simultaneous quantitation of collagen and elastin crosslinks. J. Chromatogr. A 2016, 1478, 60-67. [CrossRef]

66. Available online: http://kb.mtc-usa.com/article/AA-02558/0/ (accessed on 5 May 2019).

67. Available online: http://kb.mtc-usa.com/article/AA-02185/0/ (accessed on 5 May 2019).

68. Pesek, J.J.; Matyska, M.T.; Watanabe, S.; Makhanov, M.; Lopez, A.; Alejo, K.; Orozco, D.; Doan, L. Evaluation of silica hydride materials for the LC-MS analysis of cathinones and benzylpiperazines. Forensic Chem. 2018, 8, 90-94. [CrossRef]

69. Available online: http://kb.mtc-usa.com/article/AA-01493/0/ (accessed on 5 May 2019).

(C) 2019 by the authors. Licensee MDPI, Basel, Switzerland. This article is an open access article distributed under the terms and conditions of the Creative Commons Attribution (CC BY) license (http://creativecommons.org/licenses/by/4.0/). 